\title{
Nighttime meridional neutral wind responses to SAPS simulated by the TIEGCM: A universal time effect
}

\author{
KeDeng Zhang ${ }^{1}$, Hui Wang ${ }^{1 *}$, WenBin Wang ${ }^{2}$, Jing Liu' ${ }^{2}$, ShunRong Zhang ${ }^{3}$, and Cheng Sheng \\ 'Department of Space Physics, School of Electronic Information, Wuhan University, Wuhan 430072, China; \\ ${ }^{2}$ High Altitude Observatory, National Center for Atmospheric Research, Boulder 80301, Colorado, USA; \\ ${ }^{3}$ Haystack Observatory, Massachusetts Institute of Technology, Westford 01886, Massachusetts, USA; \\ ${ }^{4}$ Department of Physics, University of Texas at Arlington, Arlington 76013, Texas, USA
}

Key Points:

- Nighttime poleward wind changes induced by SAPS exhibit remarkable UT variations, with a maximum at 06 UT and a minimum at 00 UT.

- The UT variation of the poleward wind changes is caused by the combined effects of pressure gradient, ion drag force and Coriolis force.

- The ion drag effects on the poleward wind changes result from the displacement between the geomagnetic and geographic poles.

Citation: Zhang, K. D., Wang, H., Wang, W. B., Liu, J., Zhang, S. R. and Sheng, C. (2021). Nighttime meridional neutral wind responses to SAPS simulated by the TIEGCM: A universal time effect. Earth Planet. Phys., 5(1), 52-62. http://doi.org/10.26464/epp2021004

\begin{abstract}
The present work uses the Thermosphere lonosphere Electrodynamics General Circulation Model (TIEGCM), under geomagnetically disturbed conditions that are closely related to the southward interplanetary magnetic field (IMF), to investigate how the nighttime poleward wind $\left(30^{\circ}-50^{\circ}\right.$ magnetic latitude and 19-22 magnetic local time) responds to subauroral polarization streams (SAPS) that commence at different universal times (UTs). The SAPS effects on the poleward winds show a remarkable UT variation, with weaker magnitudes at 00 and 12 UT than at 06 and 18 UT. The strongest poleward wind emerges when SAPS commence at 06 UT, and the weakest poleward wind develops when SAPS occur at 00 UT. A diagnostic analysis of model results shows that the pressure gradient is more prominent for the developing of the poleward wind at 00 and 12 UT. Meanwhile, the effect of ion drag is important in the modulation of the poleward wind velocity at 06 and 18 UT. This is caused by the misalignment of the geomagnetic and geographic coordinate systems, resulting in a large component of ion drag in the geographically northward (southward) direction due to channel orientation of the SAPS at 06 and 18 UT ( 00 and 12 UT). The Coriolis force effect induced by westward winds maximizes (minimizes) when SAPS commence at 12 UT (00 UT). The centrifugal force due to the accelerated westward winds shows similar UT variations as the Coriolis force, but with an opposite effect.
\end{abstract}

Keywords: Subauroral Polarization Streams; TIEGCM; poleward winds; Universal time changes

\section{Introduction}

The thermospheric meridional wind is one of the keys to understanding the variability of the coupled lonosphere-Thermosphere (IT) system. It can influence the ionosphere by moving the plasma along the magnetic field lines (Rishbeth, 1967), by the ionospheric wind dynamo (Blanc and Richmond, 1980), and by other ion-neutral interaction processes (Richmond et al., 1992; Zhang SR et al., 2017), including the flywheel effect (Lyons et al., 1985; Odom et al., 1997; Richmond et al., 2003; Ridley, 2005; Deng $Y$ et al., 2014). Thus, understanding the spatial distribution of thermospheric winds is critical to elucidating the dynamics in the IT system and thus to improvement of near-Earth space environ-

Correspondence to: H. Wang, h.wang@whu.edu.cn

Received 20 MAY 2020; Accepted 28 AUG 2020.

Accepted article online 12 OCT 2020.

C 2021 by Earth and Planetary Physics. ment modeling and forecast capabilities.

At subauroral latitudes, Subauroral Polarization Streams (SAPS) are an important and interesting physical phenomenon. SAPS are described as westward plasma jets (PJ, Galperin et al., 1974) or subauroral ion drifts (SAID, Anderson et al., 1993). They are located in a latitudinally narrow region and are observed from dusk to early morning. The magnitude of the drift speed of SAPS is greater than $500 \mathrm{~m} / \mathrm{s}$ in the pre-midnight sector (Foster and Vo, 2002). SAPS are driven by the strong poleward electric field during geomagnetically disturbed periods, closely related to southward interplanetary magnetic field (IMF) $B_{z}$ conditions (Anderson et al., 1991; Yeh et al., 1991; Foster and Vo, 2002; He F et al., 2017). SAPS have substantial effects on the $F$ region zonal winds. As reported by Wang WB et al. (2012), an empirical model of SAPS was imposed in the Thermosphere lonosphere Electrodynamics General Circulation Model (SAPS-TIEGCM) to simulate SAPS effects on the IT system during a period of geomagnetically moderate activ- 
ity. Their results showed that SAPS could drive large changes globally - not just in the auroral and subauroral regions - in neutral temperature, electron density, and horizontal wind.. There are notable ion drag effects in which the neutrals are driven westward by the strong westward ion flow in the SAPS channel, producing significant temperature changes due to the ion-neutral interaction, i.e. frictional heating (Wang WB et al., 2012). Thus, in the afternoon to midnight sector, an extra strong westward wind channel is generated, which has been studied extensively by using satellite observations (e.g., Emmert et al., 2001; Wang $\mathrm{H}$ et al., 2008, 2012, 2018).

Apart from SAPS effects on the zonal winds, effects of SAPS on the thermospheric meridional winds have also been the subject of a few studies (e.g., Zhang SR et al., 2015, 2017; Guo JP et al., 2018; Ferdousi et al., 2019). Due to the lack of global meridional wind observations from satellites, the meridional winds have been studied mostly by using ground-based Fabry-Perot interferometers (FPI) observations and numerical simulations. During periods of magnetic disturbance, the energy and momentum carried by the solar wind are deposited into the IT system. Such deposition into the auroral regions of impulsive magnetosphere energy and momentum triggers travelling atmospheric disturbances (TADs) (e.g., Bruinsma and Forbes, 2007; Lei JH et al., 2008; Liu J et al., 2010, 2015, 2016). Richmond (1978) suggested that the TADs could push the ionosphere upward or downward along the magnetic field lines by the neutral meridional winds and could transport energy from high to low latitude regions. The TADs in the thermospheric meridional winds can produce disturbance dynamo electric fields in the ionosphere (Blanc and Richmond. 1980). The disturbance electric field can, in turn, directly influence the plasma and contribute to the TADs (e.g., Fujiwara and Miyoshi, 2006). This is the process of the well-known equatorward wind disturbances during storm times. By using observations from three FPI stations at mid-latitudes, Zhang SR et al. (2015) found a strong poleward surge of meridional winds at geographic middle latitudes during the 2015 St. Patrick's Day storm. The nighttime poleward wind disturbances at 20-21 local time (LT), located at $\sim 45^{\circ}$ magnetic latitude (Mat), were found to be opposite to the well-known equatorward wind disturbances that travel from high to low latitudes under geomagnetic disturbed conditions. This observation was attributed to the important role of the Coriolis force in the subauroral region. They proposed that the strong westward wind jet associated with SAPS might trigger a meridional wind change due to the Coriolis force (Zhang et al., 2015) and centrifugal force (Lühr et al., 2007). Based on Global lonosphere-Thermosphere Model (GITM) simulations, Guo JP et al. (2018) investigated theoretically the ion-neutral coupling processes induced by SAPS. In their study, the poleward surge of winds was revealed to originate from combined effects from the pressure gradient associated with the auroral energy and high-latitude convection, and the Coriolis force related to the westward neutral wind jet driven by SAPS.

While the effects of SAPS on thermospheric winds have been established in those abovementioned earlier studies and appear very straightforward, the exact wind response - particularly, the magnitude of changes in both zonal and meridional components is subject to large variability. This complexity arises from the fact that the thermospheric winds result from the balance of forces from many driving factors, including pressure gradient, Coriolis, centrifugal, ion drag, and others. The additional ion drag, as well as pressure gradient changes due to SAPS effects, modify the winds in general; in particular, they alter the ways in which the wind (zonal and meridional) components couple. Largely owing to the separation between geomagnetic and geographic poles, substantial longitudinal thermosphere and ionosphere variations exist as a result of longitudinal variations in some of these abovementioned forcings, e.g., pressure gradients caused by solar irradiation global distribution, auroral heating, and local heating associated with SAPS. Therefore UT variations in the responses of thermospheric winds to SAPS are really within expectations; however, delineation of the relative importance among those competing forcings responsible for longitudinal/UT variations remains a matter of significant scientific interest. Using GITM simulations, Wang $\mathrm{H}$ et al. (2018) investigated the zonal wind response to SAPS that occurred at different UTs. They found that the westward wind disturbances exhibited a significant UT variation; the disturbances reached their maxima (minima) at 18 (04) UT and 04 (16) UT in the Northern and Southern Hemisphere, respectively. Thus, SAPS commencing at different UTs might affect the nighttime poleward winds differently and induce UT variations in the poleward winds. This phenomenon and its associated physical mechanism (the relative importance of abovementioned forcings), however, are still poorly understood, which are thus the focus of the present work. This investigation can also contribute to understanding of the energy and momentum propagation from the mid-latitude to the equatorial region.

In the following section, we make a comparison between TIEGCM simulations and FPI observations during the 2015 St. Patrick's storm to show that the default TIEGCM without SAPS and the SAPS-TIEGCM are suitable for our study. Then we carry out a theoretical study of the UT variation of poleward winds in response to SAPS. To explore possible mechanisms driving this response, we perform a term analysis of the poleward winds.

\section{TIEGCM}

The TIEGCM is a first principles model of the coupled thermosphere and ionosphere, developed by the High Altitude Observatory of the National Center for Atmosphere Research. The TIEGCM is driven by a high-latitude electric field (Heelis et al., 1982; Weimer, 2005), solar extreme ultraviolet and ultraviolet spectral fluxes parameterized by the $F_{10.7}$ index (Richards et al., 1994), and diurnal and semidiurnal tidal inputs at the low boundary (Hagan and Forbes, 2002, 2003). The horizontal resolution of the TIEGCM is $2.5^{\circ}$ in geographic latitude (GLat) by $2.5^{\circ}$ in geographic longitude (GLon). The vertical grid is pressure coordinates with a resolution of a quarter scale height, extending from $\sim 97$ to $\sim 700 \mathrm{~km}$, depending on solar activity. For the purpose of comparing simulation with observations, the model is driven by true geophysical conditions. For idealized modeling studies, the model is run for 20 days to reach a diurnally reproducible steady state; the results are then used for the analysis. In this work (TIEGCM v2.0 is used), all model simulations have been done for two cases, one with SAPS, the other without. Thus, the differences between these two simulations are the global IT responses to SAPS. In this work, the 
electric field model at high latitudes uses the Heelis model (Heelis et al., 1982). The characteristics of the auroral particle precipitation number flux used in this TIEGCM is from Roble and Ridley, (1987). In a way similar to that of Wang WB et al. (2012), the SAPS ion velocity is imposed into the subauroral region at all altitudes in the TIEGCM, with the location and magnitude determined by the $K p$ index (SAPS-TIEGCM). The larger the $K p$, the lower is the MLat and the faster is the ion velocity of SAPS. The background $E \times B$ drift at subauroral region is replaced by the imposed SAPS ion velocity, introducing the SAPS effects into the IT system without any other changes. Thus, the global IT system in the SAPS-TIEGCM is self-consistent.

\section{Results}

\subsection{Data-Model Comparison}

The zonal ion drift velocities calculated by the SAPS-TIEGCM and the TIEGCM at 00 UT on 18 March 2015 are given in Figures 1a and $1 \mathrm{~b}$, respectively. The black lines and arrows over-plotted are the orbit and the observed ion drift velocity from the DMSP F18 satellite at 23 UT on 17 March. In Figure 1a, a notable two-cell plasma convection is present. The zonal ion velocity is strongly westward in the SAPS channel ( $60^{\circ}$ MLat and 14-22 Magnetic local time $(\mathrm{MLT})$ ), with a speed of $\sim 1200 \mathrm{~m} / \mathrm{s}$, which corresponds to the SAPS. Another peak of the westward ion velocity $(\sim 500 \mathrm{~m} / \mathrm{s})$ is located in the auroral region and dawn sector. The eastward peaks of the ion velocity occur in two sectors; one is at $\sim 70^{\circ}$ MLat and 00-09 MLT, and the other is at noon in the auroral region, with a maximum speed of $\sim 600 \mathrm{~m} / \mathrm{s}$. There is a large model-observation discrepancy in zonal ion velocity at $\sim 75^{\circ}$ MLAT and $\sim 20 \mathrm{MLT}$ : the modeled (westward) velocity is opposite to the observation (eastward). This difference does not compromise the reliability of model, however, because the pattern of subauroral zonal ion velocity is similar between the model and observation. The pattern of zonal ion velocity modeled by the TIEGCM (Figure 1b) is similar to that of the SAPS-TIEGCM (Figure 1a), with differences mainly in the SAPS channel. The TIEGCM ion velocity in the SAPS channel is small, but westward. In Figures 1a and 1b, the DMSP-observed ion velocity is westward at $\sim 60^{\circ}$ MLat and $19.5 \mathrm{MLT}$ with a speed of $\sim 1500 \mathrm{~m} / \mathrm{s}$, as shown by the black arrows. The DMSP-observed SAPS are reasonably consistent with the ones modeled in the SAPS-TIEGCM, but with small differences. For instance, the observed ion velocity at $\sim 56^{\circ}$ MLat and $08 \mathrm{MLT}$ is eastward, whereas the simulated one is westward. Such small differences are understandable and acceptable in modeling work, because the TIEGCM and the SAPS-TIEGCM are driven by the 3-hrs $K p$ index, which might cause differences between model simulations and observations when real geomagnetic activity is changing rapidly with time.

Figure 1c presents wind data from FPI observations and TIEGCM simulations at 00-03 UT on 18 March 2015. The observed poleward winds have a maximum speed of $\sim 100 \mathrm{~m} / \mathrm{s}$ (blue, black, and red lines) at 02-03 UT. The magenta line is the SAPS-TIEGCM simulated poleward winds at $40^{\circ} \mathrm{N}$ and $80^{\circ} \mathrm{W}$, which is in the area surrounded closely by the three stations (Pisgah Astronomical Research Institute (PAR, $\left.35.1^{\circ} \mathrm{N}, 82.8^{\circ} \mathrm{W}\right)$, Urbana Atmospheric Observatory (UAO, $\left.40.1^{\circ} \mathrm{N}, 88.2^{\circ} \mathrm{W}\right)$, and Millstone Hill $\left(\mathrm{MH}, 42.6^{\circ} \mathrm{N}\right.$, $\left.71.5^{\circ} \mathrm{W}\right)$ ). The poleward winds in magenta line have a maximum value of $\sim 110 \mathrm{~m} / \mathrm{s}$ at $\sim 01: 40$ UT. The peak of the simulated poleward winds occurs $\sim 50$ minutes ahead of the observed peak, which is related to two factors. One is the poleward winds generated by SAPS at almost all UTs. The other is the wind circulation (poleward at dayside and equatorward at nightside) during storm time at the dusk sector. The time differences could be due to the strong effects of SAPS. Such discrepancies are acceptable in model work, because the large-scale patterns of poleward winds are well reproduced by the SAPS-TIEGCM. At 00-02 UT, the observed poleward winds from FPI vary from station to station. At UAO, the wind is poleward while at $\mathrm{MH}$ and PAO the wind is equatorward. The modeled winds are directed poleward, consistent with the observed ones at UAO. The poleward winds due to SAPS have a maximum speed of $\sim 28 \mathrm{~m} / \mathrm{s}$ at 02:40 UT (figures not shown). Thus, the modeled poleward wind induced by SAPS amounts to $~ 30 \%$
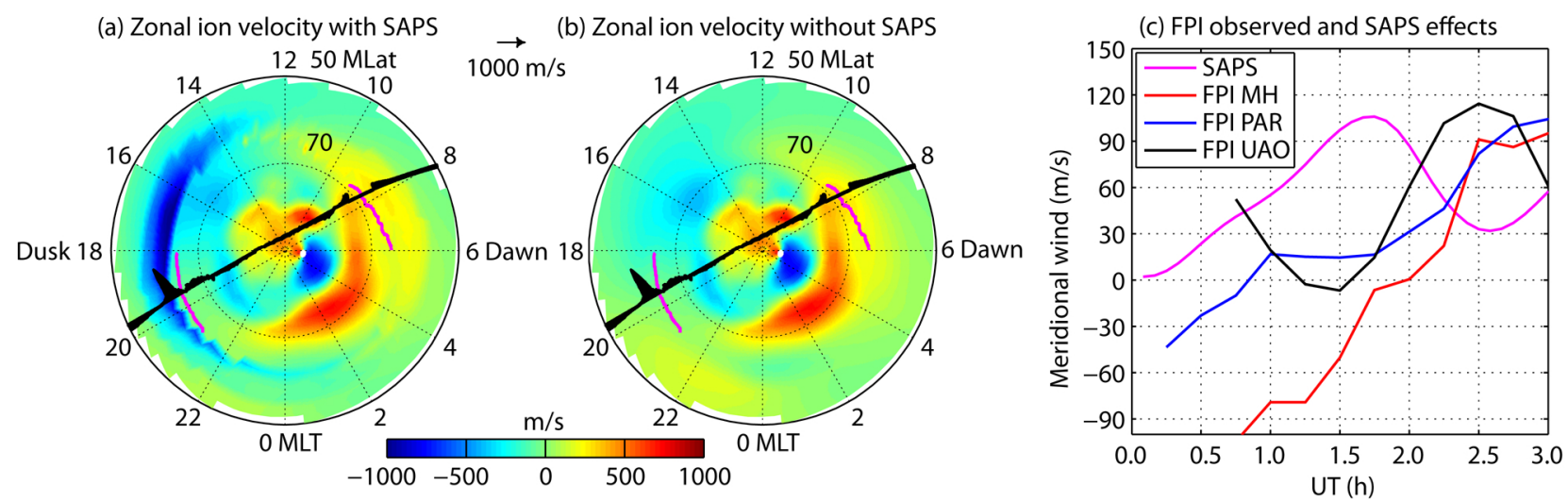

Figure 1. (a-b) Zonal ion velocity from the SAPS-TIEGCM and the TIEGCM at $250 \mathrm{~km}$ (pressure level: 0.8750) and 00:00 UT on 18 March 2015. Positive indicates the eastward direction. The black lines and arrows overplotted are the orbit and the observed horizontal cross-track ion velocity from the DMSP F18 satellite. The magenta lines overplotted are the observed auroral boundary from DMSP F18 (data products from Special Sensor Ultraviolet Spectrographic Imager, https://ssusi.jhuapl.edu). (c) Blue, black, and red lines are FPI-observed poleward winds at the Pisgah Astronomical Research Institute (PAR, 35. $\left.1^{\circ} \mathrm{N}, 82.8^{\circ} \mathrm{W}\right)$, Urbana Atmospheric Observatory (UAO, $\left.40.1^{\circ} \mathrm{N}, 88.2^{\circ} \mathrm{W}\right)$, and Millstone Hill $\left(\mathrm{MH}, 42.6^{\circ} \mathrm{N}\right.$, $71.5^{\circ} \mathrm{W}$ ) stations at 00-03 UT and $\sim 250 \mathrm{~km}$ (pressure level: 0.8750 ), respectively. The magenta line indicates the TIEGCM-simulated poleward winds at $40^{\circ} \mathrm{N}$ and $80^{\circ} \mathrm{W}$. The speed is given in $\mathrm{m} / \mathrm{s}$. 
of the observed poleward winds. The underestimation of the winds seems to be a common problem in the models. For example, Guo JP et al. (2018) showed that the meridional wind output from the global ionosphere and thermosphere model (GITM) is lower than the observed winds. The longitudinal differences in the TIEGCM zonal winds in the middle and equatorial region were reported to be lower than in the CHAMP observations (Zhang KD et al., 2019), which is due to two reasons. The first is the coarse resolution of the model ( 2.5 degree resolution in MLat), although we have used the model's highest available resolution. The second reason is that the SAPS-TIEGCM is driven by the 3-hrs $K p$ index, which is different from the realistic high-latitude driver. However, the model with SAPS imposed can generate poleward winds in a qualitative way at middle latitudes at nighttime, which can guarantee the reliability and stability of the following study.

\subsection{UT Variations of Poleward Winds Driven by SAPS}

What differences are possible in the nighttime poleward wind when SAPS occur at different UTs? To answer this question, we used the SAPS-TIEGCM to perform four idealized numerical experiments. All the results are thus for idealized cases, not for such a real case as the one shown in Figure 1. The reason idealized cases are used in the following study, instead of a realistic case, is that external driving (e.g., IMF and geomagnetic activity) at different UTs varies significantly in the realistic case. In the idealized case, we can hold the external conditions unchanged and vary only the UT time of occurrence of SAPS.

Figure 2a shows the epoch time variation of the $K p$ index, which is the input used to drive the model. The TIEGCM run without SAPS is called the "base run" hereafter. The $K p$ index begins to increase from 3 at 00:00 epoch time to 7.5 at 01:30 epoch time, then decreases to 3 at 03:00 epoch time to represent the disturbed period. The $K p$ configuration is used to identify SAPS effects on the poleward winds. The 00:00 and 03:00 epoch times mark the onset and end time of SAPS. SAPS have been included in the SAPSTIEGCM at four different UT times $(00,06,12$, and 18 UT). By differencing the base run from the four SAPS-TIEGCM runs, we can determine the effects of SAPS on the poleward wind disturbances at different UTs. In these four cases, SAPS shift equatorward with increasing $K p$ index, within the band of $40^{\circ}-55^{\circ}$ MLat and 13$24 \mathrm{MLT}$. The peak velocity $(\sim 1078 \mathrm{~m} / \mathrm{s})$ occurs at $45^{\circ}$ MLat and 19-20 MLT (01:30 epoch time). The strength of the contour SAPS channel varies with $K p$ index. That is, SAPS at larger $K p$ could pro- (a) Kp configuration

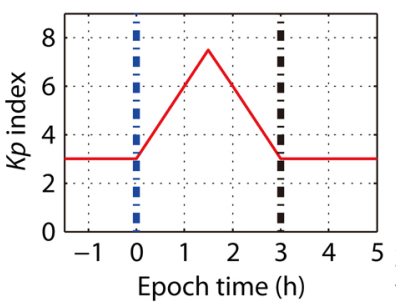

(e) epoch 1:20

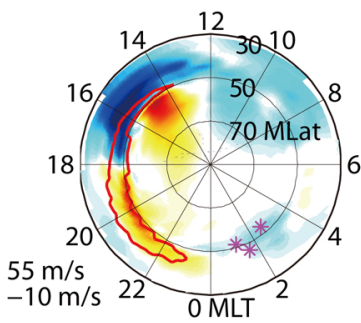

(i) epoch 2:40

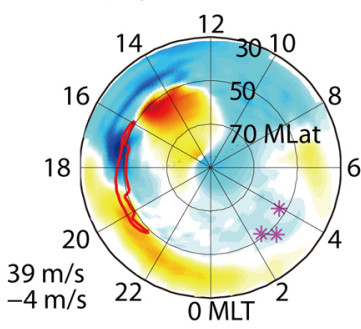

VN nh TIEGCM DSAPS 06 UT

(b) epoch 0:20

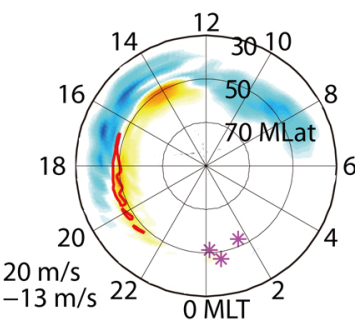

(f) epoch 1:40

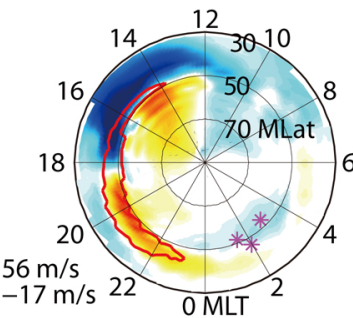

(j) epoch 3:0

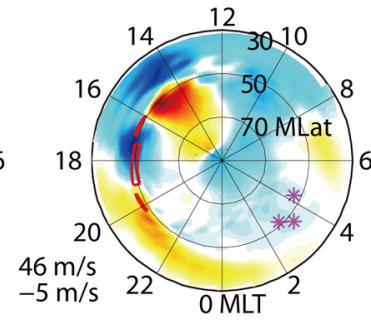

(c) epoch 0:40

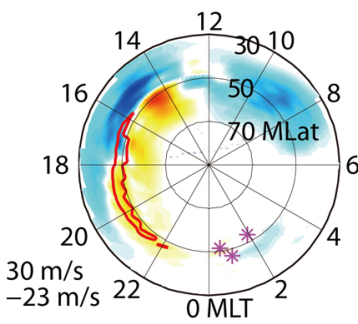

(g) epoch 2:0

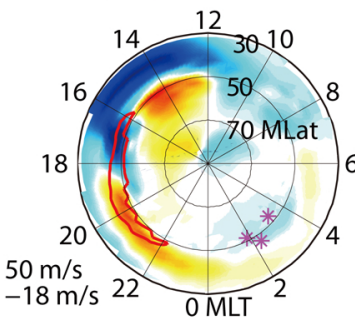

(k) epoch 3:20

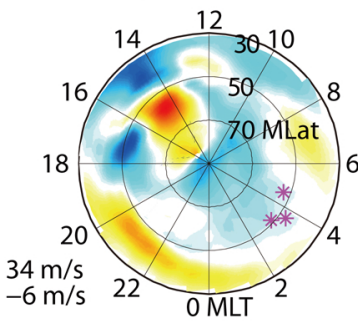

(d) epoch 1:0

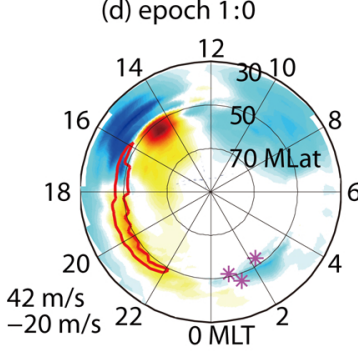

(h) epoch 2:20

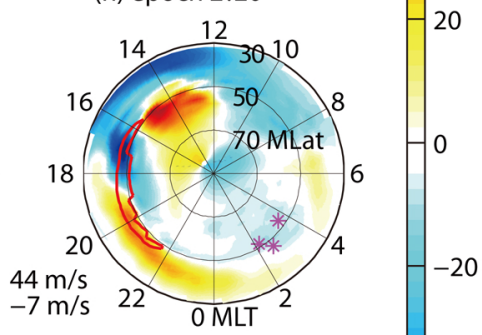

(I) epoch 3:40

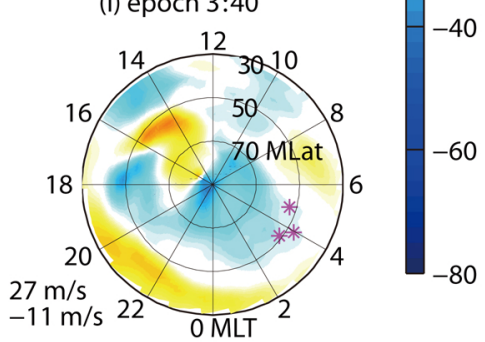

$(\mathrm{m} / \mathrm{s})$

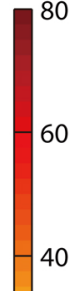

40

20

$-20$

$-40$

Figure 2. (a) The Kp index input in the TIEGCM-SAPS. The vertical dashed lines in blue and black are the onset and end time of the SAPS, respectively. (b-l) The magnetic local time and latitude variations of the geographic poleward wind responses to SAPS that commence at 06 UT. The epoch time varies from 00:20 to 03:40, with a cadence of 20 minutes. The red solid line denotes the location of SAPS. The altitude is $250 \mathrm{~km}$, and pressure level is 0.8750 . The velocity is given in $\mathrm{m} / \mathrm{s}$. The velocity at the left lower corner of each subfigure is the peak value of the equatorward (negative) and poleward (positive) winds at $30^{\circ}-50^{\circ} \mathrm{MLat}$ and $19-22 \mathrm{MLT}$. Three magenta stars, from low to high latitudes, indicate the Pisgah Astronomical Research Institute (PAR, 35. $\left.{ }^{\circ} \mathrm{N}, 82.8^{\circ} \mathrm{W}\right)$, Urbana Atmospheric Observatory (UAO, 40.1 $\left.{ }^{\circ} \mathrm{N}, 88.2^{\circ} \mathrm{W}\right)$, and Millstone Hill (MH, $42.6^{\circ} \mathrm{N}, 71.5^{\circ} \mathrm{W}$ ) FPI stations, respectively. 
duce lower MLat and faster ion velocity.

As an example, Figures $2 b-21$ show the thermospheric poleward wind disturbances in response to the SAPS that commences at 06 UT, when the nighttime meridional wind disturbances in poleward at $30^{\circ}-50^{\circ} \mathrm{MLat}$ are the strongest. The epoch time interval from 00:20 to 03:40 is shown, with a time cadence of $20 \mathrm{~min}$. In the SAPS region (enclosed by the red contour lines), the zonal wind has higher velocity and mainly directs westward at a speed of $\sim 200 \mathrm{~m} / \mathrm{s}$ (see the 3rd column of Figure 5), which is consistent with previous work (e.g. Wang $\mathrm{H}$ et al., 2008, 2012, 2018). In Figure $2 \mathrm{~b}$, the SAPS indicated by the red lines locate at $\sim 47^{\circ} \mathrm{MLat}$ and 17-21 MLT. In Figure $2 \mathrm{e}$, the SAPS appear at $40^{\circ}-50^{\circ}$ MLat and 14-23 MLT. Thereafter, they move poleward to $\sim 48^{\circ}$ MLat and sector of 16-20.5 MLT in Figure 2i (epoch time 02:40).

The poleward winds under study are enhanced as the time progresses. At 00:20 epoch time, the poleward wind disturbances at $30^{\circ}-50^{\circ}$ MLat and 19-22 MLT have an average speed of $\sim 15 \mathrm{~m} / \mathrm{s}$ (Figure $2 \mathrm{~b}$ ), with a peak speed $(\sim 20 \mathrm{~m} / \mathrm{s}$ ) occurring at $\sim 20 \mathrm{MLT}$. The peak velocity of poleward winds due to SAPS is given at the left lower corner of subfigures. Note here that the changes in the thermosphere-ionosphere coupling system are global in scale (Wang WB et al., 2012), not limited only to the region of this study's focus. The peak speed of the poleward wind changes from epoch time 00:00 to 03:00, and occurs at epoch time 01:40 (Figure 2f) and $\sim 21 \mathrm{MLT}$, with a magnitude of $\sim 56 \mathrm{~m} / \mathrm{s}$. Thereafter, it decreases with the epoch time and shifts continuously towards the pre-midnight sector. At epoch time 02:20-03:40, the large poleward wind at $19-22 \mathrm{MLT}$ and $30^{\circ}-50^{\circ}$ MLat seems to occur at relatively lower latitudes, equatorward of the SAPS channel. Note that we are interested in this paper only in the nighttime poleward wind changes induced by SAPS. In Figures $2 b-2 i$, we can see that there are also large poleward winds between 14 and 16 MLT at $\sim 60^{\circ}$ MLat. The cause and variation of these winds are of great interest, and will be investigated in a separate paper. The phenomenon that a spread band of poleward winds occurs at midlatitudes and 22-02 MLT sector, which is related to the molecular viscosity, the inertia force, and Coriolis effects due to westward winds. Based on observations and model outputs, previous studies (Fig. 6 in Wang H et al., 2011, Fig. 7 in Wang WB et al., 2012) have disclosed that the latitudes of neutral wind with a strong magnitude induced by SAPS are much greater than the latitudes of the SAPS. This difference is related to the molecular viscosity in the thermospheric composition. Furthermore, as disclosed in Wang $\mathrm{H}$ et al. (2018), the westward winds at sector of 12-24 MLT due to SAPS will not disappear at the time that the SAPS close. On the contrary, the winds will last several hours because of inertial forces, owing to the dense thermospheric density (Wang $\mathrm{H}$ et al., 2018). Thus, the poleward winds at midnight can be attributed to the Coriolis force due to westward winds (Walterscheid and Crowley, 2015).

A large equatorward wind is generated by SAPS at $30^{\circ}-50^{\circ} \mathrm{MLat}$ and 12-18 MLT in Figures $2 \mathrm{~b}-2 \mathrm{~g}$. The potential drivers might be the combined effects of the pressure gradient and ion drag. At the afternoon sector, the geomagnetic field configuration and SAPS channel orientation cause the ion drag to have a large component in the geographically southward direction; thus ion drag can have deep influences on the equatorward winds. At lower latitudes and 12-18 MLT, the pressure gradient due to the frictional heating, which heats neutrals and ions locally, can transport the heated air to lower latitudes through nonlinear dynamic processes. The equatorward winds due to SAPS at $30^{\circ}-50^{\circ} \mathrm{MLat}$ and 19-22 MLT in Figure $2 b$ have a maximum speed of $13 \mathrm{~m} / \mathrm{s}$. The peak equatorward winds spread from $19 \mathrm{MLT}$ to $21 \mathrm{MLT}$ at epoch time 00:00-02:00. During the remainder of that epoch interval, the equatorward wind disturbances induced by SAPS disappear. The potential drivers of these equatorward winds might be similar to those operating at $30^{\circ}-50^{\circ}$ MLat and 12-16 MLT, under the combined influence of ion drag and pressure gradient.

Figure 3 gives the epoch time variations of poleward wind changes caused by SAPS in the Northern Hemisphere at different UTs (see the four videos in the supporting material). The global structure of poleward wind changes in Figure $3 \mathrm{a}$ is similar to that in Figure $2 \mathrm{~b}$. The meridional winds due to SAPS at 19-22 MLT and $30^{\circ}-50^{\circ} \mathrm{MLat}$ are equatorward, with a maximum speed of $32 \mathrm{~m} / \mathrm{s}$. In Figure $3 \mathrm{~b}$, the meridional wind changes at 19-22 MLT and $30^{\circ}-50^{\circ} \mathrm{MLat}$ include the largest SAPS-influenced poleward winds - a magnitude of $\sim 25 \mathrm{~m} / \mathrm{s}$. At the focus region, a strong equatorward wind exists at lower latitudes, with a speed of $\sim 45 \mathrm{~m} / \mathrm{s}$. Note that the selected epoch times in the middle column of Figure 3 capture the maximum poleward winds at the focus region. At the epoch time marking the end of SAPS, the nightside poleward winds show a speed of $\sim 25 \mathrm{~m} / \mathrm{s}$ (19-22 MLT and $30^{\circ}-50^{\circ}$ MLat). A comparison between Figures $3 \mathrm{~d}-3 \mathrm{i}$ (poleward wind changes at 12 and $18 \mathrm{UT}$ ) and $3 a-3 c$ shows that there exist similar global structures of poleward wind changes, especially at 19-22 MLT, but with different magnitudes. The maximum magnitude is about 28 and $44 \mathrm{~m} / \mathrm{s}$ at 12 and 18 UT, respectively. Thus, the poleward wind variations at $19-22 \mathrm{MLT}$ and $30^{\circ}-50^{\circ}$ MLat are stronger at 06 and 18 UT than those at 00 and 12 UT.

\section{Discussion}

UT variations of thermospheric and ionospheric parameters (i.e., electron density, neutral winds) have been explored in recent years (e.g., Häusler et al., 2007; Cnossen and Richmond, 2012; Maute et al., 2012; Su FF et al., 2015; Li Z et al., 2018; Yang CY et al., 2018). As these studies have suggested, UT variations in the IT system are associated with effects arising from the displacement of the geomagnetic pole from the Earth's rotation axis, from the longitudinal difference of geomagnetic field, as well as from the solar illumination. The longitudinal variations of the magnetic field strength are significantly different in the two hemispheres. A onepeak longitudinal pattern appears in the Northern Hemisphere but a two-peak structure in the Southern Hemisphere (Yue J et al., 2013; Perlongo and Ridley, 2016; Laundal et al., 2017). These structures rotate with UT throughout the day. Thus, a significant longitudinal difference exists in the geomagnetic field configuration. Based on GITM simulations, Wang $\mathrm{H}$ et al. (2018) explored the disturbed zonal winds induced by SAPS at different UTs, which are induced by the ion drag effects due to the UT variations of sunlight at geomagnetic mid-latitudes. Previous studies have focused mainly on UT effects of SAPS on the zonal winds (e.g., Wang $\mathrm{H}$ et al., 2012). Studies on UT effects of SAPS on the poleward winds have been still lacking. The present work has revealed pole- 

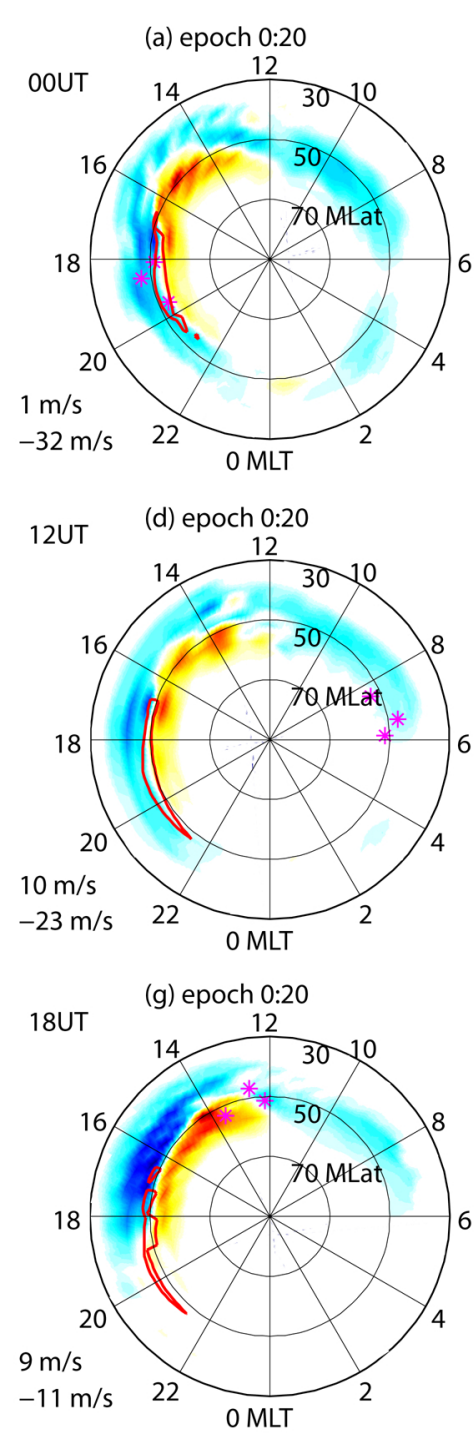
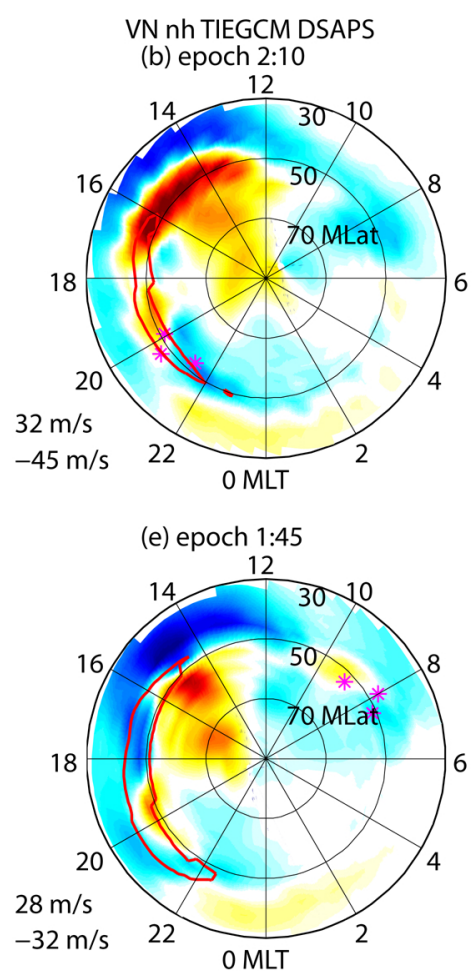

(h) epoch 2:0

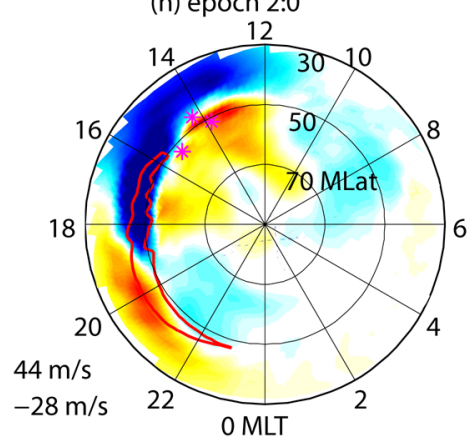

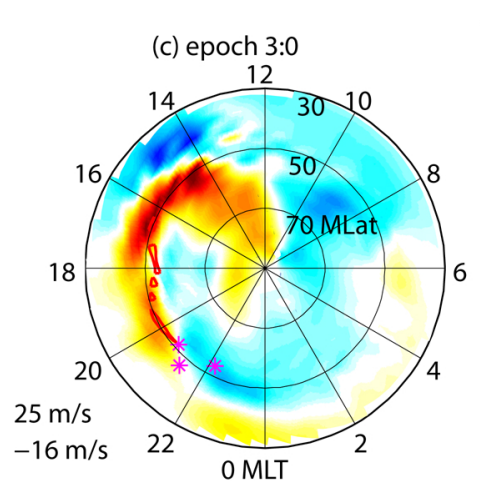
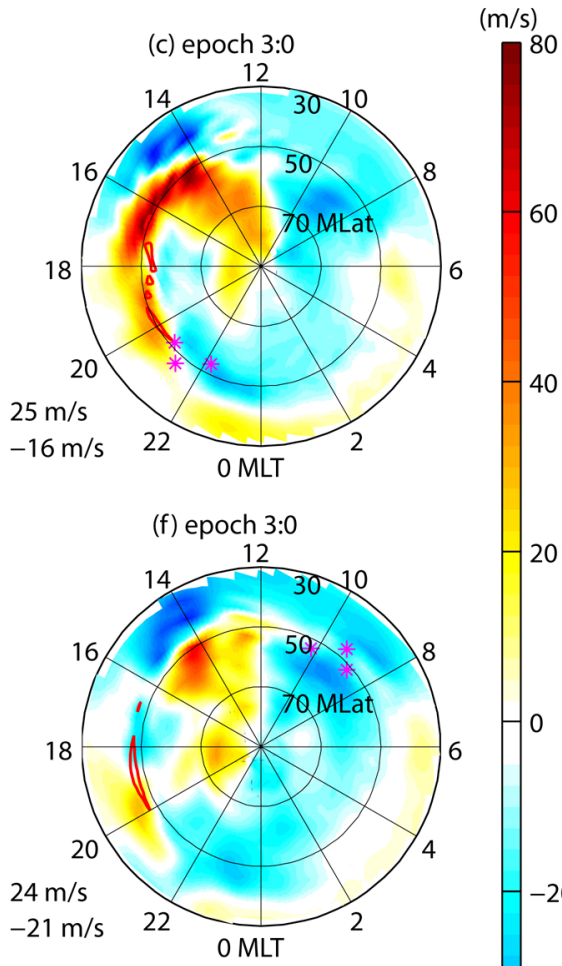

$-20$

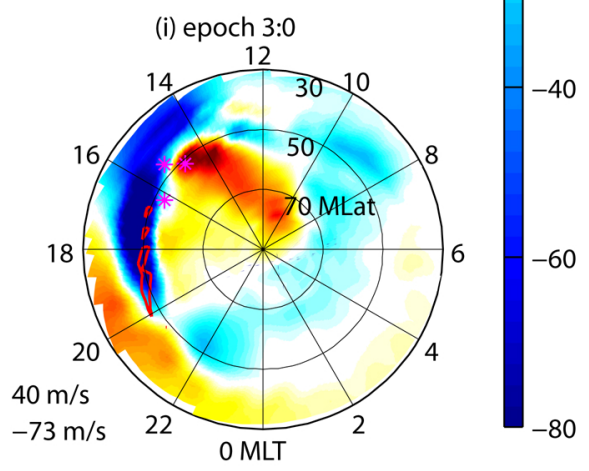

Figure 3. The magnetic local time and latitude variations of the geographic poleward wind responses to SAPS that commence at 00 (top, a-c), 12 (middle, $\mathrm{d}-\mathrm{f}$ ), and 18 (bottom, $\mathrm{g}$-i) UT. The red solid line denotes the location of SAPS. The altitude is $\sim 250 \mathrm{~km}$, and pressure level is 0.8750 . The velocity is given in $\mathrm{m} / \mathrm{s}$. The velocity at the left lower corner of each subfigure is the peak value of the equatorward (negative) and poleward (positive) winds at $30^{\circ}-50^{\circ} \mathrm{MLat}$ and $19-22 \mathrm{MLT}$. The three magenta stars from low to high latitudes indicate the PAR, UAO, and MH FPI stations, respectively.

ward wind disturbances during the nighttime in response to SAPS.

It is known that the thermospheric winds are determined by a balance among various forcing terms, including pressure gradient, ion drag, the Coriolis force, the centrifugal force, viscosity, and momentum advection (Thayer and Killeen, 1993; Richmond, 1995; Kwak and Richmond, 2007; Hsu et al., 2016; Wang H and Zhang KD, 2017; Zhang KD et al., 2018). The meridional momentum equation is

$$
\begin{aligned}
\frac{\partial v_{n}}{\partial t}= & \frac{g e^{Z}}{p_{0}} \frac{\partial}{\partial Z}\left[\frac{\mu \partial v_{n}}{H \partial Z}\right]+f^{\mathrm{cor}} u_{n} \\
& +\lambda_{y y}\left(v_{E \times B, y}-v_{n}\right)+\lambda_{y x}\left(v_{E \times B, x}-u_{n}\right)-v_{n} \cdot \nabla v_{n} \\
& +\frac{u_{n} u_{n}}{R_{\mathrm{E}}} \tan \lambda-\frac{1}{R_{\mathrm{E}}} \frac{\partial \Phi}{\partial \lambda}-W \frac{\partial v_{n}}{\partial Z}-h d_{v}
\end{aligned}
$$

where $v_{n}$ is the meridional winds, $u_{n}$ is the zonal winds, $R_{\mathrm{E}}$ is the Earth's radius, $\lambda$ is the geographic latitude, $Z$ is the altitude, $\mu$ is the molecular viscosity, and $\lambda_{y x}$ and $\lambda_{y y}$ are the ion drag coefficients. The forcing terms are, in this order: the vertical viscosity (the second term), Coriolis (the third term), ion drag (fourth and fifth terms), nonlinear horizontal advection (the sixth term), centrifugal force (the seventh term), pressure gradient (the eighth term), vertical advection (the ninth term) and horizontal diffusion (the tenth term). To investigate the mechanism of the UT dependence of SAPS effects, a term analysis in the meridional momentum equation, similar to that in Hsu et al. (2016) and Zhang KD et al. (2019), is applied to the four cases, presented in Figure 4, in which SAPS occur at different UTs. It is found that ion drag, pressure gradient, Coriolis force, and centrifugal force in the meridional momentum equation in this work are more important than the other terms considered (e.g., horizontal advection and viscos- 
ity figures not shown, Hsu et al., 2016; Zhang KD et al., 2019). Thus, ion drag, pressure gradient, Coriolis force, and centrifugal force are shown in Figure 4. Note here that the acceleration is the velocity change during unit time. Thus, the variations of acceleration do not necessarily correspond well to those of the poleward winds. A positive acceleration stands for acceleration in the poleward direction or deceleration in the equatorward direction.

In Figure 4, the sums of accelerations due to the four forcing terms are $-0.29,3.84,-0.19$, and $1.72 \mathrm{~cm} / \mathrm{s}^{2}$ at $00,06,12$, and 18 UT, respectively. Those values represent the peak values in the focus region. Additionally, through a comparison between the subfigures representative of a single force term, one can explore the detailed physical mechanisms causing the UT variations of poleward wind changes during the SAPS periods. Thus, as shown in Figures 2 and 3, the SAPS-induced poleward wind changes in the postdusk sector of (19-22 MLT) and $30^{\circ}-50^{\circ}$ MLat has the strongest magnitude when SAPS commence at 06 UT, resulting from the important role of ion drag. The weakest poleward wind changes occur around 00 UT when pressure gradient plays the prominent
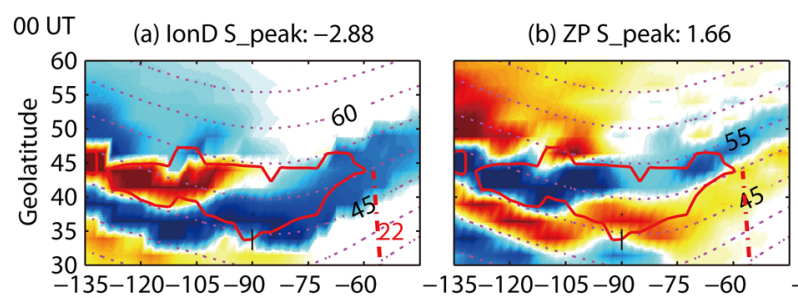

06 UT

(e) S_peak: 1.56
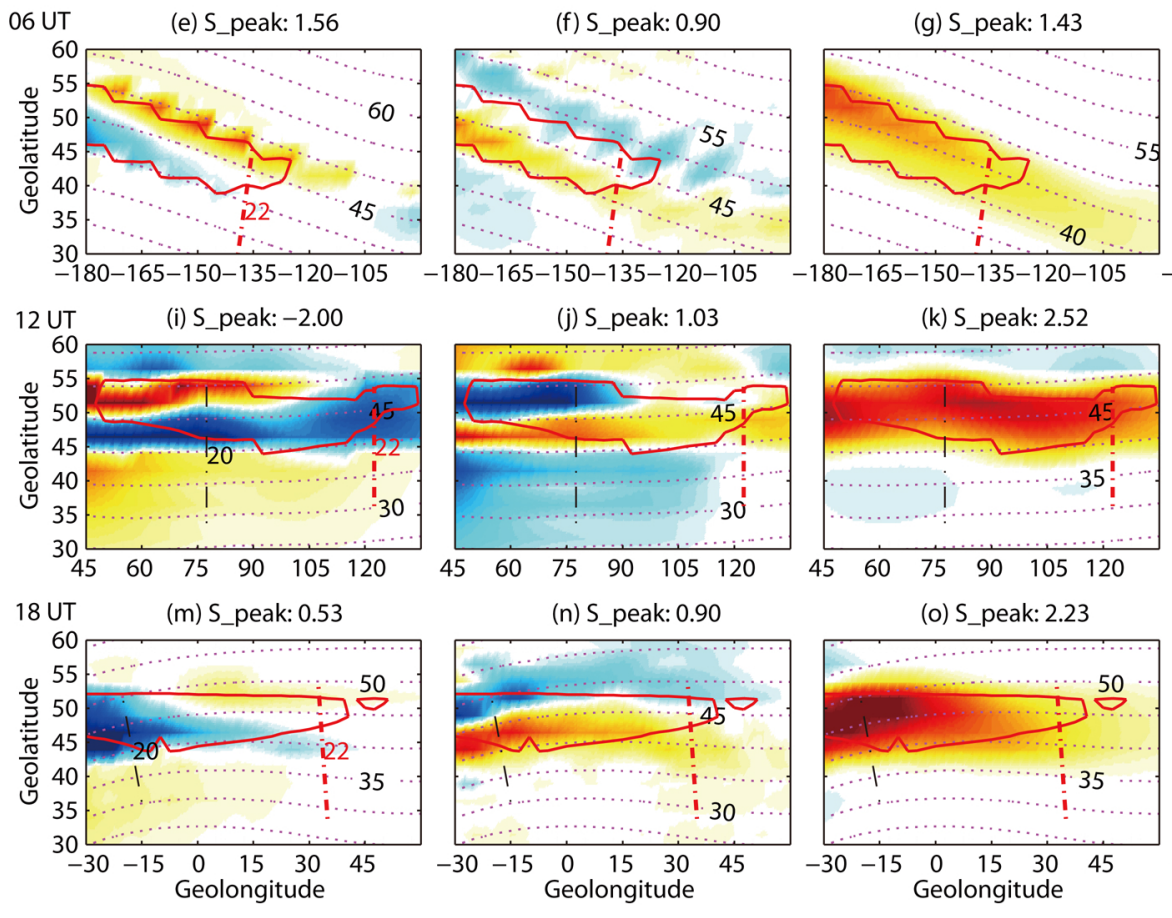

forcing role.

\subsection{Roles of Ion Drag}

The left column in Figure 4 shows the ion drag effects by SAPS at different UTs at the epoch time of peak changes of poleward winds. The magenta dash-dotted lines are the geomagnetic latitudes calculated by the International Geomagnetic Reference Field (IGRF). When SAPS occur at 06 and 18 UT (Figures $4 \mathrm{e}$ and $4 \mathrm{~m}$ ), the acceleration disturbances - the differences between with and without SAPS, due to ion drag at 19-22 MLT and $30^{\circ}-50^{\circ}$ MLat are poleward, with a maximum magnitude of 1.56 and $0.53 \mathrm{~cm} / \mathrm{s}^{2}$, respectively. Thus, ion drag contributes to the generation of poleward winds; this observation differs from the previous report of Guo JP et al. (2018). The difference may possibly be related to the location of the SAPS channel. As shown in Figure 4e, in our case the SAPS channel is aligned with geomagnetic latitude. The ion drag by SAPS is westward in the magnetic coordinates. When it is projected or viewed in geographic coordinates, it has two components, one that is geographic westward (which drives westward winds) and the other that is the geographic northward
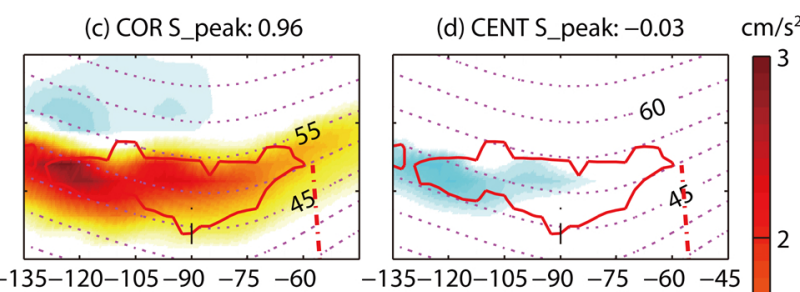

(g) S_peak: 1.43

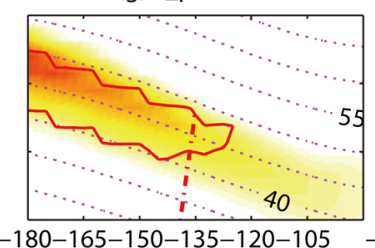

(h) S_peak: -0.05

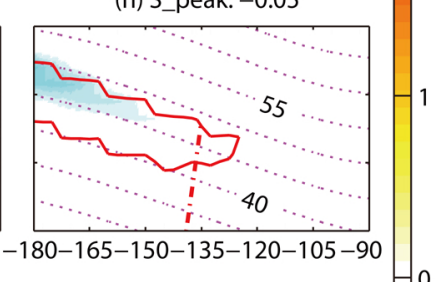

(k) S_peak: 2.52
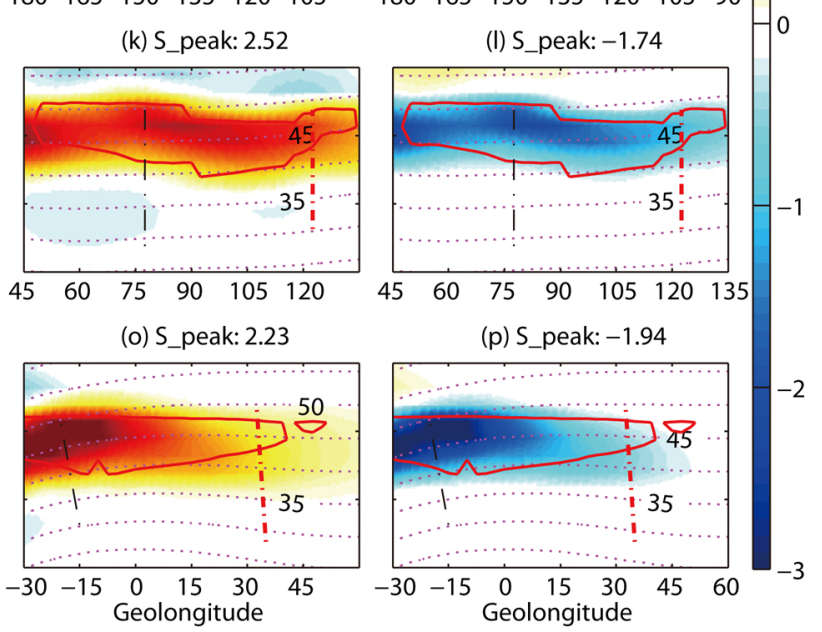

Figure 4. The geographic longitude and latitude variations of SAPS forcing terms (acceleration) in the geographic poleward direction. The top to bottom panels are for the SAPS commencing at $00,06,12$, and $18 \mathrm{UT}$, respectively. The left to right column is for the acceleration, which are the differences between with- and without-SAPS, due to ion drag (FD), pressure gradient (ZP), the Coriolis force (COR), and the centrifugal force (CENT), respectively. The magenta dash-dotted lines are the geomagnetic latitudes calculated by IGRF in the region. The red lines overplotted are the SAPS channel $(K p=7.5)$. The vertical black and red dash-dotted lines are the MLTs of 19 and $22 \mathrm{~h}$, respectively. The accelerations are given in $\mathrm{cm} / \mathrm{s}^{2}$. The S_peak is the peak value at $19-22 \mathrm{MLT}$ and $30^{\circ}-50^{\circ} \mathrm{MLat}$. A positive acceleration stands for the acceleration in the poleward direction or deceleration in the equatorward direction. 
(which contributes to poleward wind changes). As reported in the literature (Emmert et al., 2001; Wang WB et al., 2012), SAPS can drive westward neutral winds due to strong ion drag effects caused by the large relative velocity differences between the ion drifts and neutral winds. Thus, in this case, the geomagnetic field configuration and SAPS channel orientation cause the ion drag to have a large component in the geographically northward direction and, ergo, ion drag plays an important role in the generation of the poleward wind changes. On the other hand, the ion drag effects at 00 and 12 UT (Figures 4a, 4i) are different from those at 06 UT. In geographic coordinates, the SAPS are then equatorward because of the geomagnetic field configuration (red dotted lines). Therefore, the ion drag effects are southward, decelerating the poleward winds.

lon drag is proportional to ionospheric plasma density, magnetic field strength, and the velocity differences between the neutrals and ions (Richmond, 1995). There have been studies showing that both the ionosphere electron density and plasma drifts have large longitudinal variations (e.g., Maute et al., 2012; Su FF et al., 2015; Wang $\mathrm{H}$ et al., 2015; Zhong JH et al., 2016; Wang $\mathrm{H}$ and Zhang KD, 2017). Wang $H$ et al. (2018) found that the middle latitude electron densities in the subauroral region during SAPS periods are the largest at 18 UT and least at 06 UT. Under similar SAPS configurations, the ionospheric plasma background in the post-dusk sector should be different when SAPS occur at different UTs. However, the regions of interest within the SAPS channel are in the nighttime sector of 19-22 MLT. Thus, the background electron density is significantly low, and so is the ion drag effect. Thus, in the area indicated by the red lines, the ion drag effects in the geographically poleward direction should be caused mostly by the relative motion between the ions and neutrals, due to the misalignment between geomagnetic and geographic coordinate systems, as SAPS are flowing in the magnetically westward direction. Additionally, the magnetic field strength also varies with geographic longitudes, producing UT variations in the ion drag.

\subsection{Roles of Pressure Gradient}

During SAPS periods, the neutral temperature is increased due to frictional heating (Wang WB et al., 2012). Fig. 5 of Wang WB et al. (2012) shows the altitudinal profile of neutral temperature at $20 \mathrm{LT}$. In the SAPS channel region, the neutral temperature is enhanced by $\sim 50 \mathrm{~K}$. The molecular rich air is heated in the lower thermosphere and uplifted to the upper thermosphere. This is shown by the vertical winds in the middle column of our Figure 5, in this report. Temperature changes increase with altitude, since Joule heating per unit mass is larger at higher altitudes, although most of Joule heating is deposited in the E region altitude (Jee et al., 2008), causing a divergent and upward flow. The vertical winds due to SAPS are upward in the SAPS channel, but weakly downward outside the channel, with a maximum speed of 1, 4, 4, and $1 \mathrm{~m} / \mathrm{s}$ at $00,06,12$ and 18 UT within the interested area, respectively. Additionally, there is a convergent flow around the SAPS channel at $250 \mathrm{~km}$ in the middle column of our Figure 5, which is similar to Fig. 9 of Guo JP et al. (2018).

The effects of the pressure gradient are complicated, with positive and negative values, on the poleward winds; and the magnitude of the pressure gradient acceleration has notable UT vari- ations, as shown in the second column to the left of Figure 4. The acceleration are $1.66,0.9,1.03$, and $0.9 \mathrm{~cm} / \mathrm{s}^{2}$ at middle latitudes and 19-22 MLT at the epoch time of the peak poleward wind changes when SAPS commence at 00,06, 12, and 18 UT, respectively. At the equator-side (pole-side) of the SAPS channel, pressure gradient effects drive the neutrals poleward (equatorward) at 06 and at 18 UT. At 00 and 12 UT, the accelerations due to the pressure gradient are stronger. This is related to the neutral temperature enhancement caused by SAPS, which are due to the frictional heating. The left column of Figure 5 shows that the peak temperature changes in the subauroral region are $65,60,75$, and $80 \mathrm{~K}$ at $00,06,12$, and $18 \mathrm{UT}$, respectively. Because the solar illumination at fixed local times achieves its maxima at 18 UT in the Northern Hemisphere, as disclosed in Wang $\mathrm{H}$ et al. (2018), so does the background ionospheric electron density. Thus, under SAPS conditions, the plasma is more strongly accelerated, and frictional heating between the plasma and neutrals occurs (Wang WB et al., 2012; Wang $\mathrm{H}$ et al., 2018), but with a smaller magnitude, because the relative velocity differences between the ions and neutrals are small. Thus, the temperature increase at 18 UT and 00 UT is smaller, compared to increases at the other two UTs. In Figures $5 \mathrm{a}$ (00 UT) and $5 \mathrm{~g}$ (12 UT), it can be found that the peak location of temperature changes is closer and poleward to the SAPS channel than peak locations at 06 (Figure 5d) and 18 UT (Figure 5j). The temperature gradient is larger at 00 and 12 UT (Figures 5a, 5g, the green contour) than at 06 and 18 UT (Figures $5 \mathrm{~d}, 5 \mathrm{j}$ ). This difference might be the principal driver of the magnitude of the pressure gradient, causing the phenomena that the accelerations due to pressure gradient are stronger at 00 and 12 UT, but weaker at 06 and 18 UT.

\subsection{Roles of the Coriolis and Centrifugal Forces}

In the TIEGCM, the Coriolis forcing term of the poleward winds is calculated as: $f^{\text {cor }} \times u_{n}$, where $f^{\text {cor }}$ is the Coriolis force parameter and $u_{n}$ is the zonal wind. At fixed geographic latitudes, the effects of the Coriolis force on poleward winds are proportional to the zonal winds (e.g., Walterscheid and Crowley, 2015; Zhang SR et al., 2015). The Coriolis force directs poleward with a westward wind. It has UT variations and contributes to the generation of poleward wind changes during SAPS events. Due to the westward winds, at 19-22 MLT, the Coriolis force pushes the neutrals to move poleward (Zhang SR et al., 2015).

The westward wind disturbances generated by SAPS achieve their maxima at 18 UT (Wang $\mathrm{H}$ et al., 2018), with a westward speed of $279 \mathrm{~m} / \mathrm{s}$ (Figure 5l). However, in the right column of Figure 5, the maximum speeds (S_peak) of westward wind changes are about 135 (Figure 5c), 115 (Figure 5f), 181 (Figure 5i), and 169 (Figure 5l) $\mathrm{m} / \mathrm{s}$ at $00,06,12$ and $18 \mathrm{UT}$, respectively. The zone of enhanced westward wind is located at a higher geographic latitude at 06 and 12 UT. Note here that at a fixed geographic latitude, the effects of the Coriolis force are proportional to the speed of zonal winds. The effects of a given zonal wind speed are proportional to the sines of their geographic latitudes, which are larger at higher latitudes due to a larger Coriolis force coefficient. Thus, taking the zonal wind disturbances by SAPS and their geographic latitudes into consideration, the Coriolis force effects on the poleward winds are large at $12 \mathrm{UT}$, and relatively small at $00 \mathrm{UT}$, a notable 


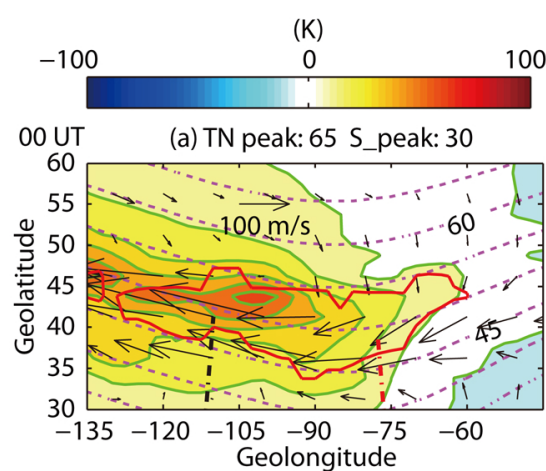

06 UT
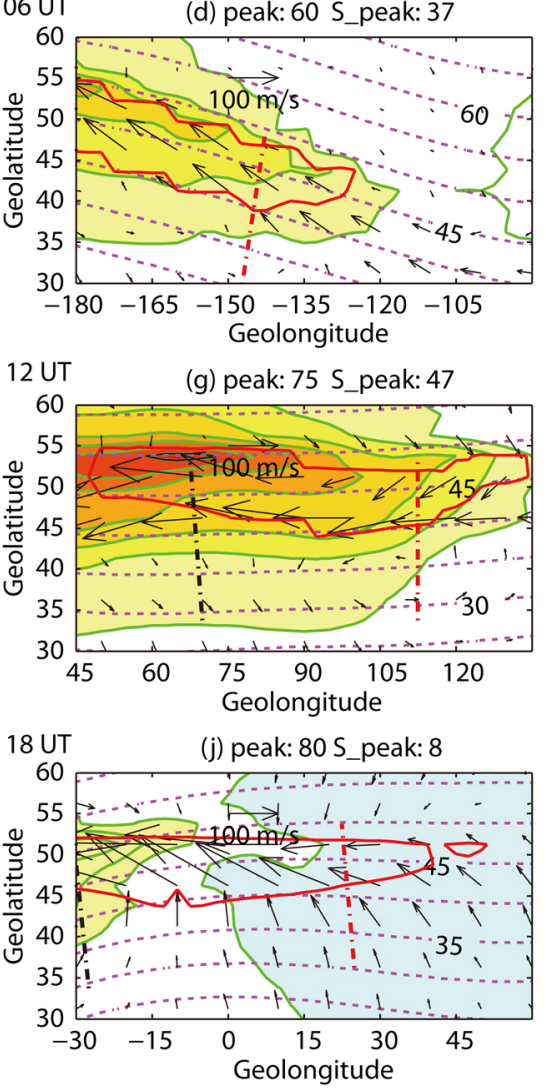

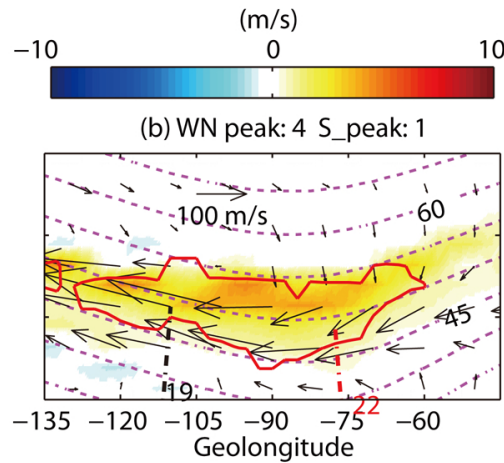

(e) peak: 6 S_peak: 4

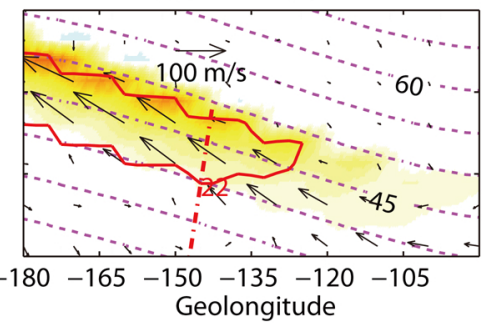

(h) peak: 5 S_peak: 4

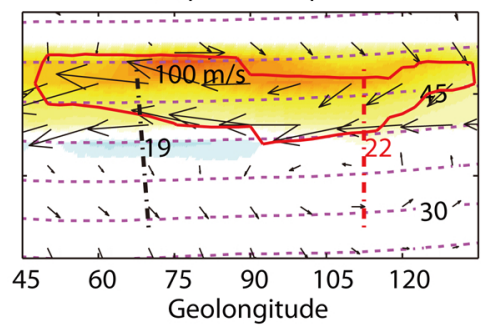

(k) peak: 6 S_peak: 1

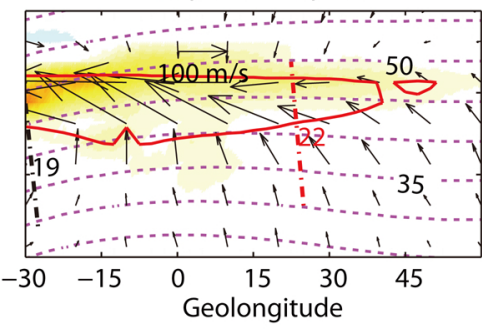

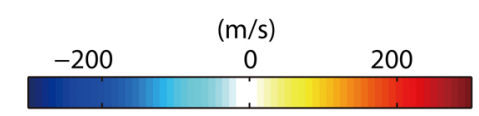

(c) UN peak: -259 S_peak: -135

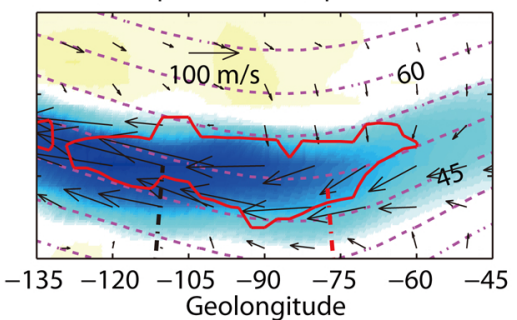

(f) peak: -229 S_peak: -115

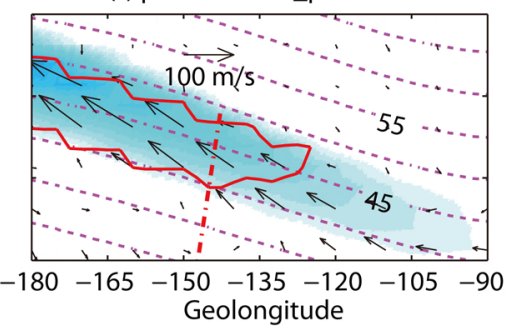

(i) peak: -214 S_peak: -181

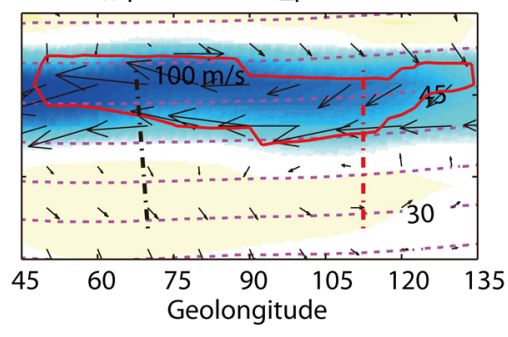

(I) peak: -279 S_peak: -169

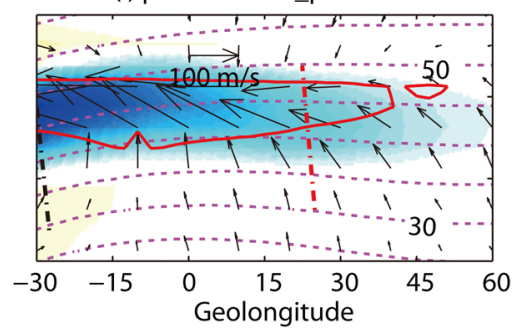

Figure 5. Similar to Figure 4, but for the neutral temperature (left), vertical (middle), and zonal wind (right) changes by SAPS. The temperature is given in $\mathrm{K}$, and the winds are given in $\mathrm{m} / \mathrm{s}$. Positive in winds denotes eastward and upward. The arrows are the horizontal wind changes by SAPS at the epoch time of peak poleward wind changes. The green contour in the left column is the neutral temperature changes by SAPS. The contour levels are the same for all UTs (temperature: $10 \mathrm{~K}$ ). The vertical black and red dash-dotted lines are the MLTs of 19 and $22 \mathrm{~h}$, respectively. The peak stands for the peak changes by SAPS globally, whereas S_peak stands for the peak changes at 19-22 MLT and $30^{\circ}-50^{\circ} \mathrm{MLat}$.

UT variation (third column to the left of Figure 4).

The poleward centrifugal force is calculated as $\frac{u_{n}^{2}}{R_{\mathrm{E}}} \times \tan \varphi$, where $R_{\mathrm{E}}$ is the Earth's radius and $\varphi$ is the geographic latitude. The centrifugal force is also proportional to the zonal winds (Lühr et al., 2007). The centrifugal force tends to drive an equatorward motion while the Coriolis force drives a northward motion with a westward wind (Lühr et al., 2007). Their contributions to the overall force balance are opposite. Similar to the pattern of Coriolis force effects is that of centrifugal force effects (see the rightmost column of Figure 4), which also exhibit UT variations that achieve their maximum $\left(-1.74 \mathrm{~cm} / \mathrm{s}^{2}\right)$ at 12 UT and minimum $\left(-0.03 \mathrm{~cm} / \mathrm{s}^{2}\right)$ at 00 UT. Thus, during periods of SAPS, the centrifugal force also contributes to the generation of poleward wind changes at different UTs. However, despite their similarities, there are also differences; for instance, the Coriolis force is strong at $00 \mathrm{UT}$, but the centrifugal force is small at the same UT, although westward zonal winds are strong at this UT (see Figure $5 \mathrm{c}$ ).

\section{Summary}

In this work, the nighttime poleward wind responses to SAPS in the Northern Hemisphere and their UT variations were explored using the TIEGCM and the SAPS-TIEGCM. We report several interesting results, as follows:

(1) Changes in the nighttime poleward wind in response to SAPS exhibit notable UT variations. The overall response is stronger at 
06 UT and weaker at 00 UT. The generation of poleward winds comes from the combined contributions of the pressure gradient, ion drag, the Coriolis force, and the centrifugal force. The pressure gradient and Coriolis force effects are strongly poleward at 00 and 12 UT, while the ion drag force is vital to the generation of the poleward wind at 06 and 18 UT. In addition, the acceleration due to the centrifugal force is weakly equatorward at 00 and 06 UT, and strongly equatorward at other UTs.

(2) SAPS are westwardly aligned in geomagnetic coordinates. Due to the misalignment between the geomagnetic and geographic coordinates, the ion drag effects of SAPS have two components in the geographic coordinates. One is the geographic westward, which drives westward winds; the other is the geographic northward (southward), which contributes to poleward wind changes at 06 and 18 UT (00 and 12 UT).

(3) The pressure gradient, in association with changes of the neutral temperature gradient induced by SAPS-related frictional heating, plays a vital role in the nighttime poleward winds. The temperature gradient is larger at 00 and 12 UT than at 06 and 18 UT; this difference accounts for the pressure gradient effects.

(4) The Coriolis force contributes to the nighttime poleward wind change at all UTs, with notable UT variations. It is stronger at 00 , 12 , and 18 UTs, and weaker at 06 UT, because of the combined effects of the geographic latitudes of the SAPS channel and the speed of zonal wind disturbances caused by SAPS.

\section{Acknowledgments}

The DMSP observations are obtained from the Madrigal database (http://cedar.openmadrigal.org/index.html/). Madrigal database is sponsored by the US NSF through a cooperative agreement (AGS1762141) with the MIT Haystack Observatory. The FPI winds are from the Madrigal database (Zhang SR et al., 2015). The simulation data are archived in NCAR supercomputer (https://www2. cisl.ucar.edu/resources/storage-and-file-systems/hpss). The $\mathrm{Na}$ tional Center for Atmospheric Research is sponsored by the $\mathrm{Na}$ tional Science Foundation. The work is supported by the National Nature Science Foundation of China (No.s 41974182, 41674153, 41521063, 41431073, 41521062 and 42004135). Research at MIT Haystack Observatory is sponsored by the US NSF award AGS1762141 and the US AFOSR MURI award FA9559-16-1-0364. This work is also supported in part by the NSF grant AGS-1452309. The Spark Project at Wuhan University (2042020gf0024) also sponsors this work.

\section{References}

Anderson, P. C., Heelis, R. A., and Hanson, W. B. (1991). The ionospheric signatures of rapid subauroral ion drifts. J. Geophys. Res.: Space Phys., 96(A4), 5785-5792. https://doi.org/10.1029/90JA02651

Anderson, P. C., Hanson, W. B., Heelis, R. A., Craven, J. D., Baker, D. N., and Frank, L. A. (1993). A proposed production model of rapid subauroral ion drifts and their relationship to substorm evolution. J. Geophys. Res.: Space Phys., 98(A4), 6069-6078. https://doi.org/10.1029/92JA01975

Blanc, M., and Richmond, A. D. (1980). The ionospheric disturbance dynamo. J. Geophys. Res.: Space Phys., 85(A4), 1669-1686.

https://doi.org/10.1029/JA085iA04p01669

Bruinsma, S. L., and Forbes, J. M. (2007). Global observation of traveling atmospheric disturbances (TADs) in the thermosphere. Geophys. Res. Lett., 34(14), L14103. https://doi.org/10.1029/2007GL030243
Cnossen, I., and Richmond, A. D. (2012). How changes in the tilt angle of the geomagnetic dipole affect the coupled magnetosphere-ionospherethermosphere system. J. Geophys. Res.: Space Phys., 117(A10), A10317. https://doi.org/10.1029/2012JA018056

Deng, Y., Huang, Y. S., Wu, Q., Noto, J., Drob, D., and Kerr, R. B. (2014) Comparison of the neutral wind seasonal variation from midlatitude conjugate observations. J. Geophys. Res.: Space Phys., 119(4), 3029-3035. https://doi.org/10.1002/2013JA019716

Emmert, J. T., Fejer, B. G., Fesen, C. G., Shepherd, G. G., and Solheim, B. H. (2001). Climatology of middle- and low-latitude daytime $F$ region disturbance neutral winds measured by Wind Imaging Interferometer (WINDII). J. Geophys. Res.: Space Phys., 106(A11), 24701-24712. https://doi.org/10.1029/2000JA000372

Ferdousi, B., Nishimura, Y., Maruyama, N., and Lyons, L. R. (2019). Subauroral neutral wind driving and its feedback to SAPS during the 17 March 2013 geomagnetic storm. J. Geophys. Res.: Space Phys., 124(3), 2323-2337. https://doi.org/10.1029/2018JA026193

Foster, J. C., and Vo, H. B. (2002). Average characteristics and activity dependence of the subauroral polarization stream. J. Geophys. Res.: Space Phys., 107(A12), SIA 16-1-SIA 16-10. https://doi.org/10.1029/2002JA009409

Fujiwara, H., and Miyoshi, Y. (2006). Characteristics of the large-scale traveling atmospheric disturbances during geomagnetically quiet and disturbed periods simulated by a whole atmosphere general circulation model. Geophys. Res. Lett., 33(20), L20108. https://doi.org/10.1029/2006GL027103

Galperin, Y., Ponomarev, V. N., and Zosimova, A. G. (1974). Plasma convection in the polar ionosphere. Ann. Geophys., 30, 1-7.

Guo, J. P., Deng, Y., Zhang, D. H., Lu, Y., Sheng, C., and Zhang, S. R. (2018). The effect of subauroral polarization streams on ionosphere and thermosphere during the 2015 St. Patrick's day storm: global ionosphere-thermosphere model simulations. J. Geophys. Res.: Space Phys., 123(3), 2241-2256. https://doi.org/10.1002/2017JA024781

Hagan, M. E., and Forbes, J. M. (2002). Migrating and nonmigrating diurnal tides in the middle and upper atmosphere excited by tropospheric latent heat release. J. Geophys. Res.: Atmos., 107(D24), ACL 6-1-ACL 6-15. https://doi.org/10.1029/2001JD001236

Hagan, M. E., and Forbes, J. M. (2003). Migrating and nonmigrating semidiurnal tides in the upper atmosphere excited by tropospheric latent heat release. J. Geophys. Res.: Space Phys., 108(A2), 1062. https://doi.org/10.1029/2002JA009466

Häusler, K., Lühr, H., Rentz, S., and Köhler, W. (2007). A statistical analysis of longitudinal dependences of upper thermospheric zonal winds at dip equator latitudes derived from CHAMP. J. Atmos. Sol.-Terr. Phys., 69(12), 1419-1430. https://doi.org/10.1016/j.jastp.2007.04.004

He, F., Zhang, X. X., Wang, W. B., and Wan, W. X. (2017). Different evolution patterns of Subauroral Polarization Streams (SAPS) during intense storms and quiet time substorms. Geophys. Res. Lett., 44(21), 10796-10804. https://doi.org/10.1002/2017GL075449

Heelis, R. A., Lowell, J. K., and Spiro, R. W. (1982). A model of the high-latitude ionospheric convection pattern. J. Geophys. Res. Space Phys., 87(A8), 6339-6345. https://doi.org/10.1029/JA087iA08p06339

Hsu, V. W., Thayer, J. P., Wang, W. B., and Burns, A. (2016). New insights into the complex interplay between drag forces and its thermospheric consequences. J. Geophys. Res.: Space Phys., 121(10), 10417-10430. https://doi.org/10.1002/2016JA023058

Jee, G., Burns, A. G., Wang, W., Solomon, S. C., Schunk, R. W., Scherliess, L., Thompson, D. C., Sojka, J. J., and Zhu, L. (2008). Driving the TING model with GAIM electron densities: lonospheric effects on the thermosphere. J. Geophys. Res.: Space Phys., 113(A3), A03305. https://doi.org/10.1029/2007JA012580

Kwak, Y. S., and Richmond, A. D. (2007). An analysis of the momentum forcing in the high-latitude lower thermosphere. J. Geophys. Res.: Space Phys., 112(A1), A01306. https://doi.org/10.1029/2006JA011910

Laundal, K. M., Cnossen, I., Milan, S. E., Haaland, S. E., Coxon, J., Pedatella, N. M., Förster, M., and Reistad, J. P. (2017). North-south asymmetries in Earth's magnetic field. Space Sci. Rev., 206(1-4), 225-257. https://doi.org/10.1007/s11214-016-0273-0

Lei, J. H., Wang, W. B., Burns, A. G., Solomon, S. C., Richmond, A. D., Wiltberger, 
M., Goncharenko, L. P., Coster, A., and Reinisch, B. W. (2008). Observations and simulations of the ionospheric and thermospheric response to the December 2006 geomagnetic storm: Initial phase. J. Geophys. Res. Space Phys., 113(A1), A01314. https://doi.org/10.1029/2007JA012807

Li, Z., Knipp, D., Wang, W. B., Sheng, C., Qian, L. Y., and Flynn, S. (2018). A comparison study of NO cooling between TIMED/SABER measurements and TIEGCM simulations. J. Geophys. Res.: Space Phys., 123(10), 8714-8729. https://doi.org/10.1029/2018JA025831

Liu, J., Zhao, B., and Liu, L. (2010). Time delay and duration of ionospheric total electron content responses to geomagnetic disturbances. Ann. Geophys., 28(3), 795-805. https://doi.org/10.5194/angeo-28-795-2010

Liu, J., Nakamura, T., Liu, L. B., Wang, W. B., Balan, N., Nishiyama, T., Hairston, M. R., and Thomas, E. G. (2015). Formation of polar ionospheric tongue of ionization during minor geomagnetic disturbed conditions. J. Geophys. Res.: Space Phys., 120(8), 6860-6873. https://doi.org/10.1002/2015JA021393

Liu, J., Wang, W. B., Burns, A., Yue, X. N., Zhang, S. R., Zhang, Y. L., and Huang, C. S. (2016). Profiles of ionospheric storm-enhanced density during the 17 March 2015 great storm. J. Geophys. Res.: Space Phys., 121(1), 727-744. https://doi.org/10.1002/2015JA021832

Lühr, H., Rentz, S., Ritter, P., Liu, H., and Häusler, K. (2007). Average thermospheric wind patterns over the polar regions, as observed by CHAMP. Ann. Geophys., 25(5), 1093-1101. https://doi.org/10.5194/angeo25-1093-2007

Lyons, L. R., Killeen, T. L., and Walterscheid, R. L. (1985). The neutral wind "flywheel" as a source of quiet-time, polar-cap currents. Geophys. Res. Lett., 12(2), 101-104. https://doi.org/10.1029/GL012i002p00101

Maute, A., Richmond, A. D., and Roble, R. G. (2012). Sources of low-latitude ionospheric $\mathbf{E} \times \mathbf{B}$ drifts and their variability. J. Geophys. Res.: Space Phys., 117(A6), A06312. https://doi.org/10.1029/2011JA017502

Odom, C. D., Larsen, M. F., Christensen, A. B., Anderson, P. C., Hecht, J. H., Brinkman, D. G., Walterscheid, R. L., Lyons, L. R., Pfaff, R., and Emery, B. A. (1997). ARIA II neutral flywheel-driven field-aligned currents in the postmidnight sector of the auroral oval: A case study. J. Geophys. Res.: Space Phys., 102(A5), 9749-9759. https://doi.org/10.1029/97JA00098

Perlongo, N. J., and Ridley, A. J. (2016). Universal time effect in the response of the thermosphere to electric field changes. J. Geophys. Res.: Space Phys., 121(4), 3681-3698. https://doi.org/10.1002/2015JA021636

Richards, P. G., Fennelly, J. A., and Torr, D. G. (1994). EUVAC: A solar EUV flux model for aeronomic calculations. J. Geophys. Res.: Space Phys., 99(A5), 8981-8992. https://doi.org/10.1029/94JA00518

Richmond, A. D. (1978). Gravity wave generation, propagation, and dissipation in the thermosphere. J. Geophys. Res.: Space Phys., 83(A9), 4131-4145. https://doi.org/10.1029/JA083iA09p04131

Richmond, A. D., Ridley, E. C., and Roble, R. G. (1992). A thermosphere/ionosphere general circulation model with coupled electrodynamics. Geophys. Res. Lett., 19(6), 601-604. https://doi.org/10.1029/92GL00401

Richmond, A. D. (1995). lonospheric electrodynamics. In H. Volland (Ed.), Handbook of Atmospheric Electrodynamics (pp. 249-290). Boca Raton: CRC Press.

Richmond, A. D., Lathuillère, C., and Vennerstroem, S. (2003). Winds in the highlatitude lower thermosphere: Dependence on the interplanetary magnetic field. J. Geophys. Res.: Space Phys., 108(A2), 1066. https://doi.org/10.1029/2002JA009493

Rishbeth, H. (1967). The effect of winds on the ionospheric F2-peak. J. Atmos. Terr. Phys., 29(3), 225-238. https://doi.org/10.1016/0021-9169(67)90192-4

Ridley, A. J. (2005). A new formulation for the ionospheric cross polar cap potential including saturation effects. Ann. Geophys., 23(11), 3533-3547. https://doi.org/10.5194/angeo-23-3533-2005

Roble, R. G., \&Ridley, E. C. (1987). An auroral model for the NCAR thermospheric general circulation model (TGCM). AnGeo, 5, 369-382.

Su, F. F., Wang, W. B., Burns, A. G., Yue, X. S., and Zhu, F. Y. (2015). The correlation between electron temperature and density in the topside ionosphere during 2006-2009. J. Geophys. Res.: Space Phys., 120(12), 10724-10739. https://doi.org/10.1002/2015JA021303

Thayer, J. P., and Killeen, T. L. (1993). A kinematic analysis of the high-latitude thermospheric neutral circulation pattern. J. Geophys. Res.: Space Phys., 98(A7), 11549-11565. https://doi.org/10.1029/93JA00629

Walterscheid, R. L., and Crowley, G. (2015). Thermal cell structures in the highlatitude thermosphere induced by ion drag. J. Geophys. Res.: Space Phys., 120(8), 6837-6850. https://doi.org/10.1002/2015JA021122

Wang, H., Ridley, A. J., Lühr, H., Liemohn, M. W., and Ma, S. Y. (2008). Statistical study of the subauroral polarization stream: Its dependence on the cross-polar cap potential and subauroral conductance. J. Geophys. Res. Space Phys., 113(A12), A12311. https://doi.org/10.1029/2008JA013529

Wang, H., Lühr, H., Häusler, K., and Ritter, P. (2011). Effect of subauroral polarization streams on the thermosphere: A statistical study. J. Geophys. Res., 116(A3), A03312. https://doi.org/10.1029/2010JA016236

Wang, H., Lühr, H., and Ma, S. Y. (2012). The relation between subauroral polarization streams, westward ion fluxes, and zonal wind: Seasonal and hemispheric variations. J. Geophys. Res.: Space Phys., 117(A4), A04323. https://doi.org/10.1029/2011JA017378

Wang, H., Ridley, A. J., and Zhu, J. (2015). Theoretical study of zonal differences of electron density at midlatitudes with GITM simulation. J. Geophys. Res.: Space Phys., 120(4), 2951-2966. https://doi.org/10.1002/2014JA020790

Wang, H., and Zhang, K. D. (2017). Longitudinal structure in electron density at mid-latitudes: Upward-propagating tidal effects. Earth, Planets Space, 69(1), 11. https://doi.org/10.1186/s40623-016-0596-9

Wang, H., Zhang, K. D., Zheng, Z. C., and Ridley, A. J. (2018). The effect of subauroral polarization streams on the mid-latitude thermospheric disturbance neutral winds: a universal time effect. Ann. Geophys., 36(2), 509-525. https://doi.org/10.5194/angeo-36-509-2018

Wang, W. B., Talaat, E. R., Burns, A. G., Emery, B., Hsieh, S. Y., Lei, J. H., and Xu, J. Y. (2012). Thermosphere and ionosphere response to subauroral polarization streams (SAPS): Model simulations. J. Geophys. Res.: Space Phys., 117(A7), A07301. https://doi.org/10.1029/2012JA017656

Weimer, D. R. (2005). Improved ionospheric electrodynamic models and application to calculating Joule heating rates. J. Geophys. Res.: Space Phys., 110(A5), A05306. https://doi.org/10.1029/2004JA010884

Yang, C. Y., Smith, A. K., Li, T., and Dou, X. K. (2018). The effect of the MaddenJulian oscillation on the mesospheric migrating diurnal tide: a study using SD-WACCM. Geophys. Res. Lett., 45(10), 5105-5114. https://doi.org/10.1029/2018GL077956

Yeh, H. C., Foster, J. C., Rich, F. J., and Swider, W. (1991). Storm time electric field penetration observed at mid-latitude. J. Geophys. Res.: Space Phys., 96(A4), 5707-5721. https://doi.org/10.1029/90JA02751

Yue, J., Wang, W. B., Richmond, A. D., Liu, H. L., and Chang, L. C. (2013). Wavenumber broadening of the quasi 2 day planetary wave in the ionosphere. J. Geophys. Res.: Space Phys., 118(6), 3515-3526. https://doi.org/10.1002/jgra.50307

Zhang, K. D., Wang, W. B., Wang, H., Dang, T., Liu, J., and Wu, Q. (2018). The longitudinal variations of upper thermospheric zonal winds observed by the CHAMP satellite at low and midlatitudes. J. Geophys. Res.: Space Phys., 123(11), 9652-9668. https://doi.org/10.1029/2018JA025463

Zhang, K. D., Liu, J., Wang, W. B., and Wang, H. (2019). The effects of IMF Bz periodic oscillations on thermospheric meridional winds. J. Geophys. Res.: Space Phys., 124(7), 5800-5815. https://doi.org/10.1029/2019JA026527

Zhang, S. R., Erickson, P. J., Foster, J. C., Holt, J. M., Coster, A. J., Makela, J. J., Noto, J., Meriwether, J. W., Harding, B. J., and Kerr, R. B. (2015). Thermospheric poleward wind surge at midlatitudes during great storm intervals. Geophys. Res. Lett., 42(13), 5132-5140. https://doi.org/10.1002/2015GL064836

Zhang, S. R., Erickson, P. J., Zhang, Y. L., Wang, W. B., Huang, C. S., Coster, A. J., Holt, J. M., Foster, J. F., Sulzer, M., and Kerr, R. (2017). Observations of ionneutral coupling associated with strong electrodynamic disturbances during the 2015 St. Patrick's Day storm. J. Geophys. Res.: Space Phys., 122(1), 1314-1337. https://doi.org/10.1002/2016JA023307

Zhong, J. H., Lei, J. H., Wang, W. B., Burns, A. G., Yue, X. A., and Dou, X. K. (2016). Longitudinal variations of topside ionospheric and plasmaspheric TEC. J. Geophys. Res.: Space Phys., 122(6), 6737-6760. https://doi.org/10.1002/2017JA024191 